\title{
Using Frequency Domain Analysis Techniques for Diagnosis of Planetary Bearing Defect in a CH-46E Helicopter Aft Gearbox
}

\author{
L. Zhou, F. Duan, D. Mba, W. Wang, and S. Ojolo
}

\begin{abstract}
Condition monitoring for helicopters has always been one of the most critical technologies to guarantee the integrity of the rotorcrafts, enhance operational and personnel safety, and reduce the overall maintenance costs. Over the past decades, health and usage monitoring system (HUMS) has been developed and implemented in helicopters to monitor the health status for the main gearbox (MGB) and other key components of the transmission system, improving condition-based maintenance for helicopters. However, many studies have indicated that current HUMS has a limited sensitivity to MGB planetary bearing defects. To enhance HUMS' performance, this paper presents an approach based on frequency domain analysis techniques to diagnose planetary bearing defects using real helicopter data collected from a CH-46E helicopter aft MGB. Vibration data was processed using signal processing techniques including self-adaptive noise cancellation (SANC), discrete-random separation (DRS), cepstrum editing, kurtogram, envelope analysis and iterative envelope cancellation. Processing results conclude that frequency domain analysis techniques can provide distinct and intuitive indications of the seeded defects at both the inner race and the outer race of the faulty planetary bearing.
\end{abstract}

Keywords:

Helicopter MGB, planetary bearing fault diagnosis, signal processing

\section{Introduction}

The helicopter transmission system is vitally important for transferring power to main rotor, tail rotor and other accessory systems [1]. The transmission system mainly consists of helicopter main gearbox (MGB), tail rotor gearbox (TGB), drive shafts and other components including gears, bearings, clutches and freewheel units. A helicopter's transmission system facilitates manoeuvres that cannot be performed by a traditional fixed wing aircraft. 
Examples of such manoeuvres including vertical take-off and landing, flying backward and sideways, hovering and many others. Therefore, it is crucial to monitor the condition of helicopter transmission system and maintain an overall healthy status for the rotorcrafts, so that catastrophic failures can be prevented. For such purpose, development of helicopter health and usage monitoring system (HUMS) was initiated in early 1990s [2]. HUMS has drawn extensive attentions since then. Being implemented for almost 30 years, HUMS has been proven to be effective in reducing accidents rate and overall maintenance costs, enhancing operational safety and improving rotorcrafts airworthiness $[3,4,5]$. Nonetheless, since the first HUMS system went operational in 1991, the significance of validating HUMS and new signal processing algorithms has been emphasised numerous times [6, 7]. Moreover, recent helicopter accidents related to MGB failures $[8,9]$ and studies published by several researchers $[10,11]$ suggested that, HUMS has limited sensitivity to the detection of MGB planetary bearing faults. As such, although vibration analysis techniques for fixed shaft, simple bearing system have been relatively well developed, it is essential that effective modifications to those techniques and combination of processing procedures to be further investigated for improving HUMS capabilities to diagnose planetary bearing defects in MGB.

In this paper, vibration data collected from a CH-46E helicopter aft MGB with seeded defects formed the basis of the investigation, which explored various frequency domain signal processing techniques for fault identification. Signal processing techniques including iterative envelope cancellation, selfadaptive noise cancellation (SANC), discrete random separation (DRS) and cepstrum editing were applied to separate planetary bearing signals from overwhelming gear mesh signals and suppress excessive frequency sidebands. Kurtogram and envelope analysis were employed as benchmark methods to detect and extract hidden diagnostic information from signal envelope. Intuitive diagnosis results were acquired via signal processing with aforementioned techniques.

\section{A description of the $\mathrm{CH}-46 \mathrm{E}$ aft gearbox seeded test}

The vibration data was collected from a series of seeded defect tests in a CH-46E aft gearbox by Westland Helicopter Limited, and digitalised by NRAD (Naval Research and Development Centre) in 1993 [12]. According to the test report of this datasets [7], eight conditions were tested, including seven seeded defect conditions and a no-defect condition. Only one type of defect was introduced during each defect tests. These test conditions are listed as follows: 
- No defect

- Planetary gear bore/bearing inner race corrosion

- Spiral bevel input pinion spalling

- Helical input pinion chipping

- Collector gear cracking

- Quill shaft cracking

- Input pinion bearing corrosion

- Helical idler gear cracking

In this paper, data collected from the condition "Planetary gear bore/bearing inner race corrosion" was analysed.

There were eight "Endevco 7259A" accelerometers installed on the testrig. This type of accelerometer has a wide dynamic range from $-500 \mathrm{~g}$ to $+500 \mathrm{~g}$, and a flat frequency amplitude response to up to $50 \mathrm{kHz}$ [13]. An optical tachometer was also fitted near scavenge pump drive. All data was recorded via a 28-Channel Racal Storehorse tape recorder, which had an aggregate $2 \mathrm{MHz}$ sampling rate or throughput for all channels. Recorded data was then digitalised at a sampling frequency of $103116.08 \mathrm{~Hz}$. The installation of some of the accelerometers is presented in Figure 1.

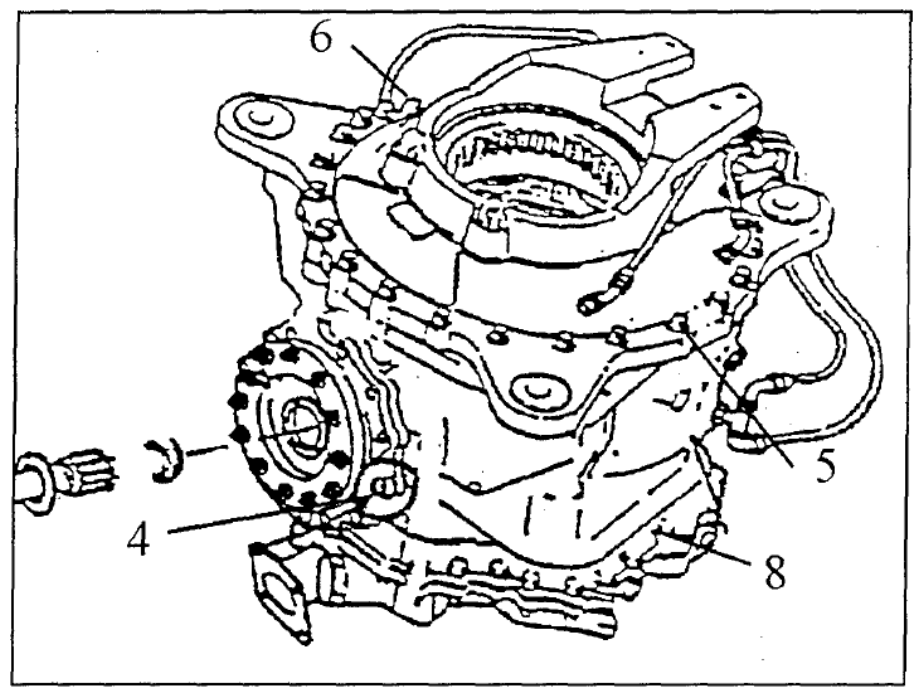

Figure 1: Sketch of installed accelerometers in CH-46 helicopter aft gearbox [12]

Vibration data from accelerometer 5 and 6 in Figure 1 were employed for this investigation (Y6 and Y7 channel respectively). These sensors were closely located to the MGB planetary speed reduction module, where the bearing corrosion defects were seeded. An optical tachometer was also employed (Y10 channel). 
Table 1 details all relevant frequency components associated with the test-rig. The planetary bearing inner race defect frequency $F_{I R D}$ and outer race defect frequency $F_{O R D}$ are calculated according to the bearings' geometry structure and rotating speed. In practice, the observed defect frequencies could have slight deviations from the calculated values, due to the randomness induced by the slip of bearing rolling elements.

Table 1: Rig related parameters

\begin{tabular}{lc}
\hline MGB input shaft speed & $324.6 \mathrm{~Hz}$ \\
MGB output shaft speed & $4.4 \mathrm{~Hz}$ \\
Sampling frequency & $103116.1 \mathrm{~Hz}$ \\
Data recording length & $21.9 \mathrm{~s}$ \\
Tachometer shaft speed & $114.4 \mathrm{~Hz}$ \\
Bearing outer race defect $F_{O R D}$ & $68.4 \mathrm{~Hz}$ \\
Bearing inner race defect frequency $F_{I R D}$ & $101.7 \mathrm{~Hz}$ \\
\hline
\end{tabular}

\section{Signal processing techniques}

\subsection{Overview of helicopter planetary bearing fault diagnosis}

Vibration-based healthy monitoring (VHM) is one of the most common monitoring techniques for rotating machines. The HUMS system also utilises VHM to monitor the health condition of the helicopters' key transmission components by collecting vibration data through installed accelerometers. A typical methodology to monitoring vibration levels is via the use of condition indicators (CIs), which are based on time series vibration data associated with gears, bearings and shafts [14]. Normally CIs have pre-determined thresholds, which generate warnings or alarms once breached. However, helicopter accidents that occurred recently $([8,9])$ indicated that HUMS failed to provide reliable and accurate fault diagnosis, especially for planetary bearing defects in the MGB. The fundamental reasons are that vibration data is susceptible to many factors including signal transmission paths, signal attenuation, machine operating noise and background noises. The other major problem is that, in relation to helicopter planetary bearing monitoring, an extra modulation of the bearing vibration signal exists as the planetary bearing is orbiting around the carrier shaft simultaneously with planetary gears, rendering CI-based algorithms less sensitive to bearing fault signatures.

Frequency-based analysis techniques are essential for extracting defectinduced repetitive signal patterns in frequency domain, therefore providing 
more indications of defects that cannot be discovered in the time domain. Specifically, for bearing fault diagnosis, demodulating structural-related high frequency resonances where bearing signals are amplified is effective in mitigating amplitude modulations, speed fluctuations and the gear meshes [15]. To enhance such processing approach, techniques can be applied to separate non-deterministic bearing signals from deterministic gear signals $[16,17,18]$. To benefit from envelope demodulation, the kurtogram was developed to identify the frequency band that contains the most impulsive signals associated with structural resonances [19, 20, 21]. Frequency-domain signal processing techniques that were implemented and evaluated in this study are introduced in the rest of this section.

\subsection{Gear and bearing signal separation techniques}

The exact planetary bearing configuration was not given in the original research report [7], therefore, a general sketch of a planetary bearing is given in Figure 2. Unlike normal bearings that are installed in simple mechanical systems, the planetary bearing in a helicopter MGB is integrated with the planetary gear whose inner bore is the outer raceway of the bearing. Therefore, when the planetary gears and rollers are rotating around the carrier shafts, inherently weak bearing signals are overwhelmed by deterministic gear meshes. However, it has been discovered that bearing signals have certain degrees of randomness due to the slip of the bearing rollers. This feature can be utilised to distinguish non-deterministic bearing signals from deterministic gear signals using adaptive filters, as deterministic signals theoretically have longer correlation length [22].

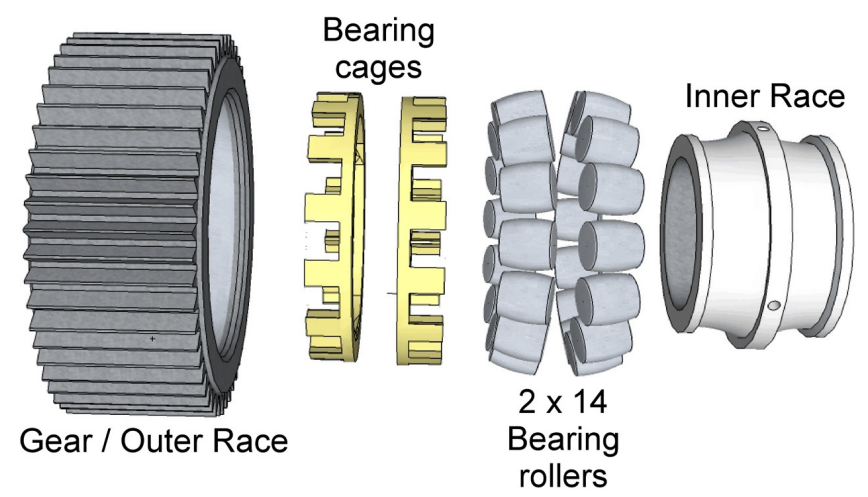

Figure 2: Sketch of planetary gear configuration in G-REDL accident report [9]

Generally, a mixed signal that consists desired signal $D(t)$ and unwanted noise $N(t)$ which is to be filtered, should be adopted as primary input $P(t)$ 
for the adaptive filter. A stand-alone noise signal $N_{0}$ that is correlated with unwanted noise $N(t)$ in primary signal should be utilised as a reference input $R(t)$. This is described in equations below.

$$
\begin{aligned}
& P(t)=D(t)+N(t) \\
& R(t)=N_{0}(t)
\end{aligned}
$$

Since reference noise signal $N_{0}(t)$ is correlated with unwanted noise $N(t)$, but not correlated with desired signal $D(t)$ in primary input, $N(t)$ can be adaptively predicted based on $N_{0}(t)$ and subtracted from $P(t)$, leaving output signal with mostly desired $D(t)$.

Based on this notion, Ho and Randall proposed separating random bearing signals and discrete gear mesh signals with an adaptive filter [23], where the reference signal is substituted by a delayed version of primary signal $P(t-\Delta t)$. This technique is called self-adaptive noise cancellation (SANC) because it does not require extra reference signals. Taking most commonly implemented least-mean-square (LMS) adaptive filtering method as an example, the SANC algorithm is demonstrated in Figure 3.

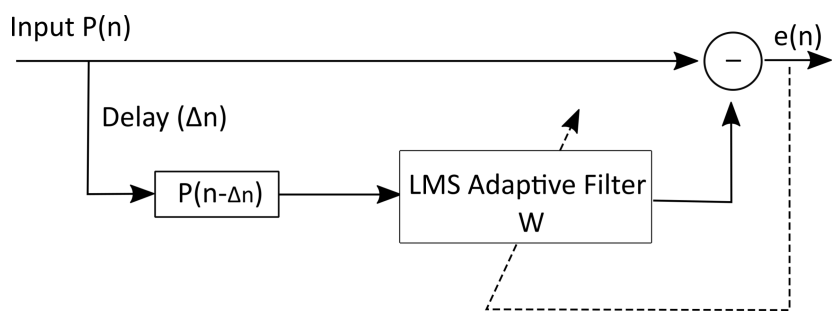

Figure 3: Schematic diagram of SANC

LMS adaptive rules are defined as [23, 24]:

$$
\begin{aligned}
e(n) & =P(n)-W_{n}^{T} \times P(n-\Delta n) \\
W_{n+1} & =W_{n}+\mu \times e(n) \times P(n-\Delta n),
\end{aligned}
$$

where $\mu$ is the convergence factor which determines the adaptation step, $e(n)$ is the output error and $W$ is the LMS filter coefficients. For a discrete time series input $P(n)$, by utilising an optimal delay $\Delta n$ when random bearing signals lose their correlations while deterministic gear meshes remain correlated, the bearing signals can no longer be accurately predicted. The output error is produced to drive the adaptation of LMS filter $W$. Through adaptive filtering, gear meshes can be consistently predicted and subtracted from $P(t)$. 
Although theoretically SANC can achieve almost complete removal of undesired gear meshes, it has been reported that finding the optimal filter length, delay $\Delta n$ and convergence factor $\mu$ can be cumbersome. Time delay affects the result of SANC directly. While a short delay has limited separation effectiveness, a delay that is too long may have the complete opposite effect. Moreover, a small convergence factor can render the algorithm unstable while a large $\mu$ provides coarse separation results.

Antoni and Randall proposed a separation technique in [18] as an adequate substitute to SANC, namely discrete random separation (DRS). DRS achieves similar or slightly compromised results compared with SANC, though for much more computational efficiency. DRS constructs the filter based on the concept of frequency response function $(F R F)$, which is achieved by blocking original data into several windowed segments to prevent frequency leakage, and then calculating $F R F$ of each block with its corresponding delays. The process is defined as:

$$
H(f)=\frac{E\left[G_{b}(f) \cdot G_{a}^{*}(f)\right]}{E\left[G_{a}(f) \cdot G_{a}^{*}(f)\right]}
$$

where $E[\cdot]$ is expectation operator. $G_{a}$ and $G_{a}^{*}$ are conjugate pairs of current windowed segment's fast Fourier transform $(F F T)$, and $G_{b}$ is its delayed signal's FFT. This averaging process is continuously conducted, to eventually produce a normalised indicator containing values from 0 to 1 , where 0 stands for index of random frequency components and 1 represents discrete frequency components. A filter thus can be developed and applied back on time series signals to filter out the unwanted discrete gear mesh signals. It has been recommended that a rule-of-thumb for selecting possible delays is about 100 periods of the centre frequency of demodulated frequency band. In practical this value could be 3 times larger [22].

DRS has been extensively discussed in [15, 22]. Multiple articles have claimed successes on applying DRS for bearing and gear signal separation $[17,25,26,27,28,29]$. It is also worth noticing that, although DRS has an advantageous processing efficiency, its performance is susceptible to varying rotating speed due to the lack of adaptive process.

\subsection{Cepstrum editing}

Cepstrum was first developed for applications in seismology and speech analysis, but it was then discovered by Randall that cepstrum analysis is competent to suppress gear-shaft related frequency sidebands efficiently with tolerance of varying rotating speed [30] . Generally, complex cepstrum is described as "the power spectrum of the logarithm of the power spectrum". Given that: 


$$
\begin{gathered}
X(f)=F[x(t)]=A(f) \cdot e^{j \phi(f)} \\
\ln [X(f)]=\ln [A(f)]+j \phi(f),
\end{gathered}
$$

where $F$ is the operator for fast Fourier transform $(F F T), X(f)$ is the Fourier transform of time domain signal $x(t), \ln$ is the natural logarithm and $A(f)$, $\phi(f)$ are amplitude and phase of $X(f)$ respectively, complex cepstrum is defined as [31]:

$$
\text { Cepstrum }=F^{-1}[\ln (X(f))]
$$

Equation 8 transforms signal in time domain firstly to frequency domain, then to "quefrency" domain. Editing components in quefrency domain, for instance, eliminating certain quefrency components will suppress all corresponding frequency components including harmonics and sidebands in frequency domain. While the phase $\phi(f)$ can be conveniently set to zero to generate the real cepstrum, it cannot be inversed back to time domain directly after being edited due to the loss of phase information. In $[32,33,31,34,30]$, researches all concluded that for bearing fault diagnosis, it is usually unnecessary to use complex cepstrum, since the editing on complex cepstrum cannot be observed directly in time domain, and the edited complex signal phase may have distortions. Thus, a modified real cepstrum editing method is advocated, which reserves the phase information from FFT, and then combines phase information with edited real cepstrum. Therefore, the edited real cepstrum can be inversed back to time domain for post-analysis.

Cepstrum editing benefits the suppression of the periodic frequency sidebands, rather than complete removal of discrete gear mesh frequencies. In [35], the authors set the entire quefrency zone to 0 except for quefrency at the very beginning whose reciprocate represents very high frequency contents. They reported that since bearing signals were not strictly periodic, they did not present strong peaks in the absolute value of the cepstrum and were not affected by the liftering. Other than this processing approach, exponential window based cepstrum editing method was proposed in [31], which essentially lifters cepstrum using an exponential window with the form of $e^{-\sigma \cdot t}$, where $\sigma$ is a time constant and should be determined by system modal information. Applying such exponential lifter can smoothly suppress high quefrency components (gear-related) and pass low quefrency components (bearing-related). In practice, it was recommended to apply cepstrum editing as a pre-processing step before other techniques that alter the composition of entire spectrum [33]. 


\subsection{Envelope analysis and iterative envelope cancellation}

Envelope analysis has been established as benchmark processing technique for bearing fault diagnosis for over 40 years [36]. Traditionally, envelope analysis requires pre-acquired knowledge of the mechanical system structural resonances. Weak bearing signals modulate the amplitude of these resonances, which in return, retains the bearing information in the resonances' envelopes. By demodulating signal in the optimal high frequency band, the fault-excited repetitive patterns can be extracted from the envelope spectrum of demodulated resonances. Hilbert transform specifically facilitates the implementation of envelope analysis, by converting signal to an analytical form, whose magnitude is original signal's envelope.

However, the effectiveness of envelope analysis is heavily dependent on the selection of the optimal structural resonances. Although such resonances are correlated with bearing geometry and can be estimated in a simple system, it becomes tremendously difficult if the target bearing is located in a complex rotational system, i.e. a helicopter gearbox. If the optimal demodulation band is unable to be discovered, it could be tedious to manually select and test every possible resonance in frequency domain.

One solution for this issue is to use a very efficient and fast-calculative algorithm, namely the iterative envelope cancellation. This algorithm was proposed recently by Ming et al. [37], which utilises iterative envelope calculations to suppress gear mesh signals in frequency domain. Their research specifically studied the effect of Hilbert transform on discrete gear mesh signals and random bearing signals. Mathematical deduction was elaborated in their study, concluding that through iteratively calculating signal squared envelope using Hilbert transform and subtracting DC components, the discrete frequency components suffer larger attenuation in amplitude, compared with that of bearing-related components. The reasons are that bearing-related signals can be modelled as a train of high frequency transient impulses, while gear meshes are periodic cumulations. Square envelope of the signal potentially introduces cross-terms between these two types of signals, weakening the pattern of periodic cumulations. The schematic diagram of iterative envelope cancellation is demonstrated in Figure 4.

In Figure 4, the stop point of the iteration is determined by control factor $\eta$, which is defined as [37]:

$$
\eta=\frac{\left|2 \times\left\langle E n v^{k-1}, E n v^{k}\right\rangle\right|}{\left\langle E n v^{k-1}, E n v^{k-1}\right\rangle+\left\langle E n v^{k}, E n v^{k}\right\rangle},
$$

where $k$ represents current iteration index, $\langle\cdot\rangle$ represents inner product operator. $\eta$ described the extent of difference between current $k$ iteration calculated signal envelope with $k-1$ iteration envelope. For instance, $\eta=1$ means 
the two envelopes are exactly the same, hence more iterations of calculation are redundant. Based on processing experiences, stop factor $\eta_{0}$ should be wisely chosen between 0.8 up to 0.95 for distinct gear mesh suppression and subsequent envelope spectrum analysis.

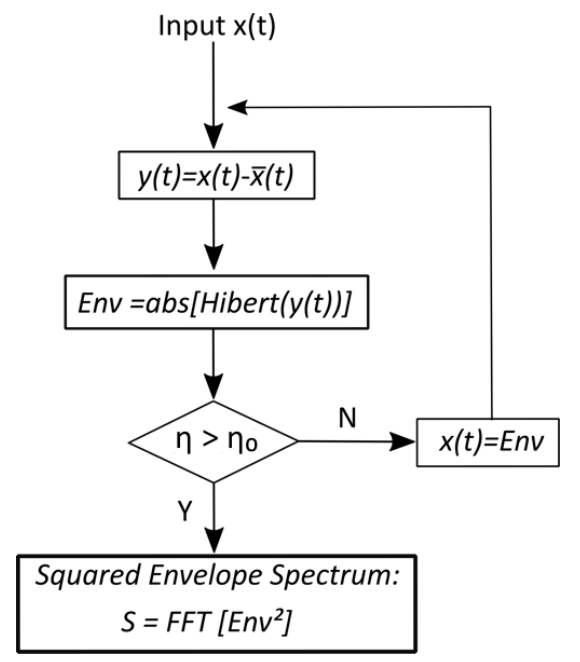

Figure 4: Schematic diagram of iterative envelope cancellation

Iterative envelope cancellation solves the problem of traditional envelope analysis by evading the selection of optimal frequency band. Instead, the application of sub-optimal frequency band is remedied by iterative calculations and high-pass the input signal at a relatively high frequency where resonance spikes are more concentrated, which only requires rough knowledge from observing frequency spectrum of input signal.

\subsection{Kurtogram}

Another method for finding the optimal demodulation frequency band is kurtogram. Kurtogram was developed by Antoni and Randall [20] from the concept of kurtosis and spectral kurtosis. Classical condition indicator kurtosis is extensively used for determining to what extent a signal is peaky. However, kurtosis becomes less sensitive as the machine condition goes severer. This issue can be addressed by examining the spectral kurtosis of signal in different frequency band $\Delta f$ with centre frequency $f_{c}$. Spectral kurtosis (SK) was first devised by Dwyer in [38] for detection of randomly occurring signals [39]. Antoni gave a detailed study of SK in $[19,20]$. By his definition, SK of a time domain signal $x(t)$ is produced from short-time Fourier transform (STFT), $X(t, f)$, which is quantitatively the local Fourier transform of original signal at time index $t$ with a sliding window moving across the entire signal record. The mathematical form of spectral kurtosis is defined as [22]: 


$$
S K(f)=\frac{E\left\{|X(t, f)|^{4}\right\}}{E\left\{\left|X(t, f)^{2}\right|\right\}^{2}}-2,
$$

where $E[\cdot]$ is the expectation operator. In order to fully examine possible combination of $\Delta f$ and $f_{c}$ for envelope demodulation, Antoni and Randall proposed a way to calculate SK not only as function of $t$ and $f$, but also as function of frequency bin $\Delta f$, namely the kurtogram. Kurtogram facilitates the application of envelope analysis by providing potential demodulation centre frequency and frequency band, which contains most impulsive signal that could be excited by bearing defects. Nonetheless, it has been reported that the indication of kurtogram is not always correct, especially for processing fault signal captured from a complex mechanical system such as a planetary gearbox [40].

\section{4. $\mathrm{CH}-46 \mathrm{E}$ helicopter data processing results}

The schematic diagram for signal processing is depicted in Figure 5. The strategy adopted to diagnose $\mathrm{CH}-46$ helicopter planetary bearing fault was firstly extracting faulty bearing related signals from the originally acquired vibration data using SANC or DRS, followed by applying iterative envelope cancellation technique. Cepstrum editing was employed as an optional step before SANC or DRS, where there were requirements to suppress excessive frequency sidebands that cannot be eliminated by separation techniques. Kurtogram combined with envelope analysis were implemented as a benchmark method for comparison with iterative envelope cancellation.

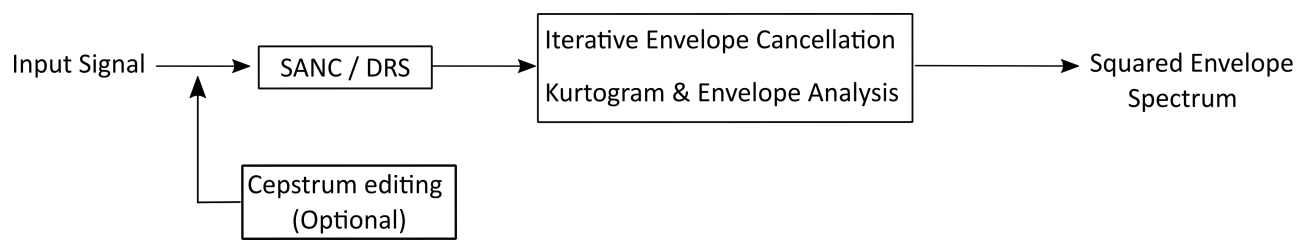

Figure 5: Schematic diagram of signal processing procedures

Basic inspection of the raw vibration data spectrum was first conducted for Y6 and Y7, as shown in Figure 6. The FFT calculation was carried out with 2260992 data points which was the data recording length, rendering $0.456 \mathrm{~Hz}$ resolution. 

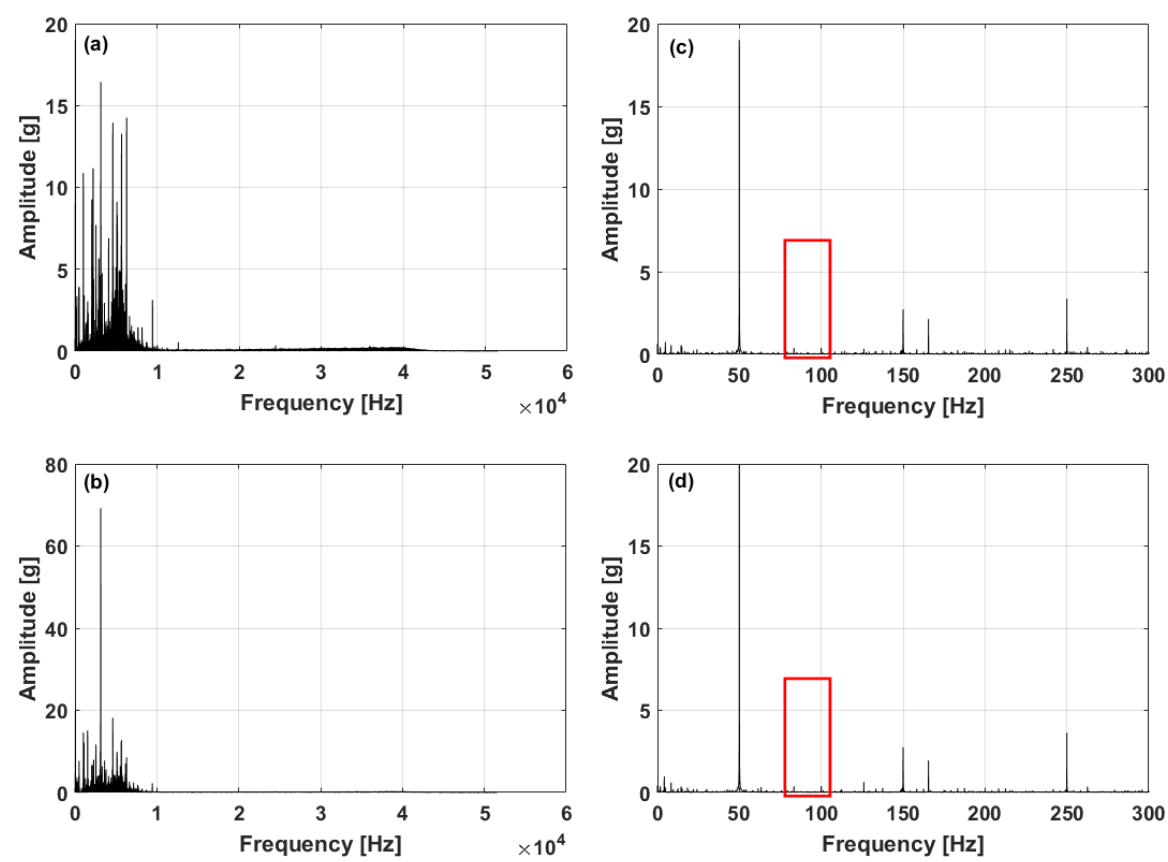

Figure 6: Amplitude spectrum of raw vibration data

(a), (c): Amplitude spectrum of Y6 and zoomed spectrum Y6

(b), (d): Amplitude spectrum of Y7 and zoomed spectrum Y7

From Figure 6, some elemental observations can be made that:

- Very limited information can be found in bandwidth higher than approximately $10 \mathrm{kHz}$. It is inferred that frequency contents higher than $10 \mathrm{kHz}$ were pre-filtered during the acquisition process.

- From zoomed spectrum, no existence of inner race or outer race defect frequency can be observed. Discrete frequency spikes that are related to gear meshes and shaft rotations are dominant in (c) and (d).

DRS and SANC were then applied to provide comparative separation results for iterative envelope cancellation. To determine the best delays for both techniques, a range of time delays from 100 to 300 periods of $10 \mathrm{kHz}$ were considered, according to the recommendations of Randall and Antoni [22] . In Figure 7, the separation using DRS for Y6 is demonstrated. The delay was selected to be 1400 points, filter length was chosen to allow 2000 times of averages to mitigate potential speed fluctuations. It is clearly shown that large discrete frequency components in Figure 7(a) have been effectively eliminated in Figure 7(b). 

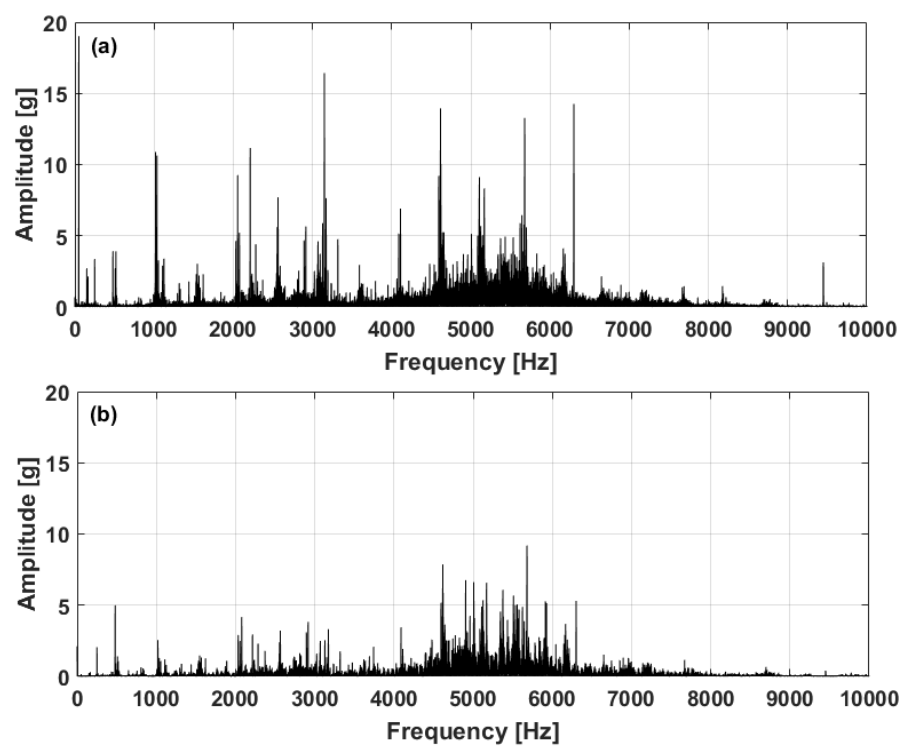

Figure 7: Demonstration of DRS separation effect for Y6, delay $=1400$

(a): Amplitude spectrum of Y6, raw data

(b): Amplitude spectrum of Y6, after DRS

The separated signals were then utilised as inputs for iterative envelope cancellation. For Y6 signal, to control the optimal output with least interferences, a $5 \mathrm{kHz}$ high-pass filter was applied to further reduce low frequency gear meshes. A stop factor $\eta_{0}$ was selected at 0.85 . The output of iterative envelope cancellation was squared envelope spectrum, which were zoomed into $0-300 \mathrm{~Hz}$ range for defect frequency identification.

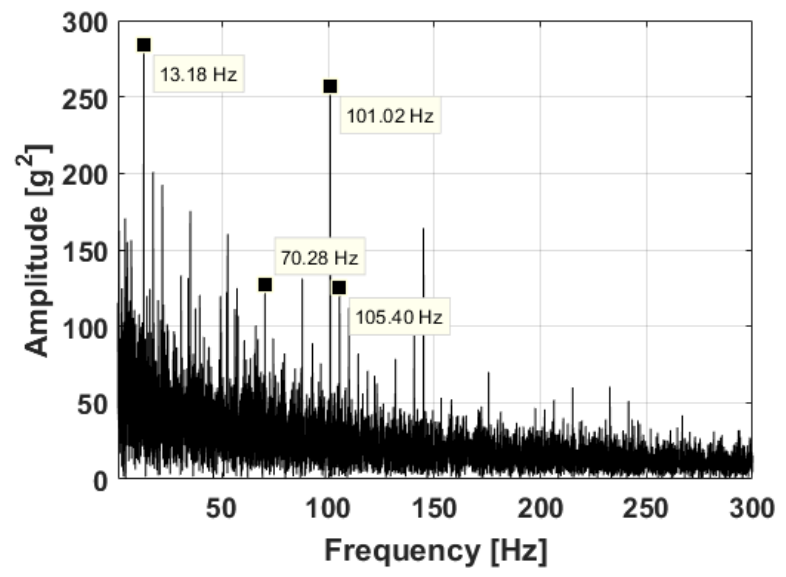

Figure 8: Squared envelope spectrum with DRS and iterative envelope cancellation, Y6 
Figure 8 clearly demonstrates the existence of the $70.28 \mathrm{~Hz}$ outer race defect frequency and the $101.02 \mathrm{~Hz}$ inner race defect frequency. The presence of $13.18 \mathrm{~Hz}$, which is 3 times the planetary carrier shaft speed $(4.4 \mathrm{~Hz})$, is associated with the planetary gear rotation speed, i.e. $4.4 \times 117 / 39=13.2 \mathrm{~Hz}$, where 117 is the number of ring gear tooth and 39 is the number of planet gear tooth. The frequency at $105.4 \mathrm{~Hz}$ was noted as a modulation sideband that resulted from $4.4 \mathrm{~Hz}$ drive shaft frequency. To further suppress unwanted frequency components, cepstrum editing was attempted to Y6 data. The real cepstrum of $\mathrm{Y} 6$ is shown in Figure 9.

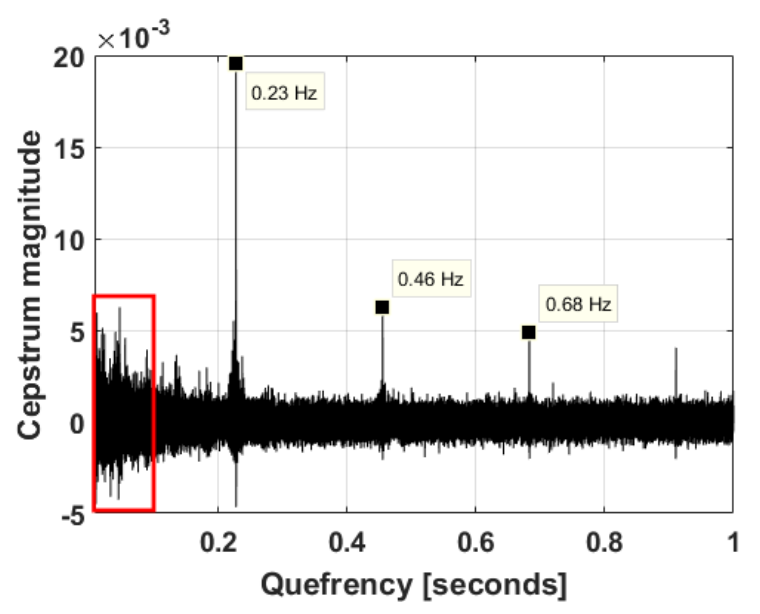

Figure 9: Real cepstrum of Y6

In Figure 9, it is evident that the 0.23 quefrency which corresponds to $4.4 \mathrm{~Hz}$ is dominant. In order to retain the characteristics of low quefrency contents and suppress high quefrency contents, a short-pass lifter was applied for all quefrency components below $1 / 114.2 \mathrm{~Hz}$. The pass region is marked in Figure 9. 


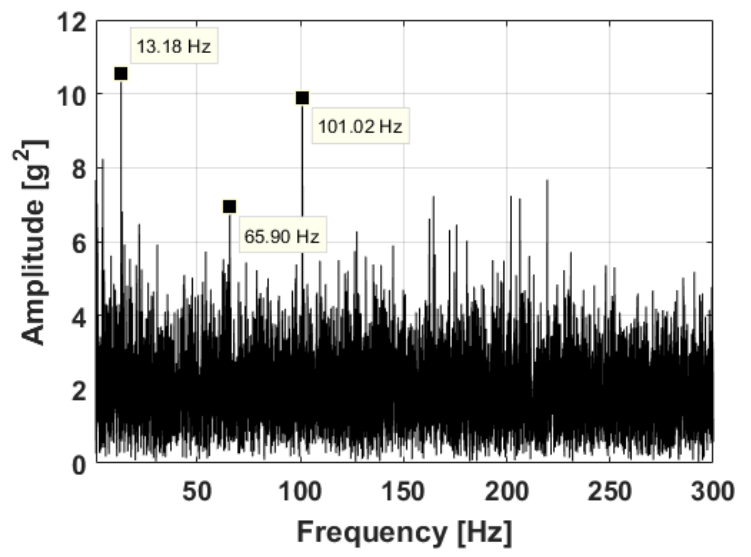

Figure 10: Squared envelope spectrum with cepstrum editing, DRS and iterative envelope cancellation, Y6

Figure 10 shows the squared envelope spectrum after cooperating shortpassed cepstrum editing. The inner race defect frequency of $F_{I R D}$ at $101.02 \mathrm{~Hz}$ is more distinct compared with that shown in Figure 8, while the outer race defect frequency $F_{O R D}$ at $70.28 \mathrm{~Hz}$ has been suppressed. However, the $65.9 \mathrm{~Hz}$ component which is a $4.4 \mathrm{~Hz}$ sideband of $F_{O R D}$ can still be observed. The other interferential sidebands have been largely suppressed.

Comparatively, the results using cepstrum editing, SANC and iterative envelope cancellation are shown in Figure 11. In Figure11(a), it is demonstrated that, using SANC with iterative envelope cancellation can reveal inner race defect frequency at $101.2 \mathrm{~Hz}$. However, only $4.4 \mathrm{~Hz}$ sideband of outer race defect frequency can be observed at $74.67 \mathrm{~Hz}$. In Figure 11(b), the result suggests that combining cepstrum editing and SANC can achieve suppression of $101.02 \mathrm{~Hz} F_{I R D}$ component, but revealing $70.27 \mathrm{~Hz} F_{O R D}$. 

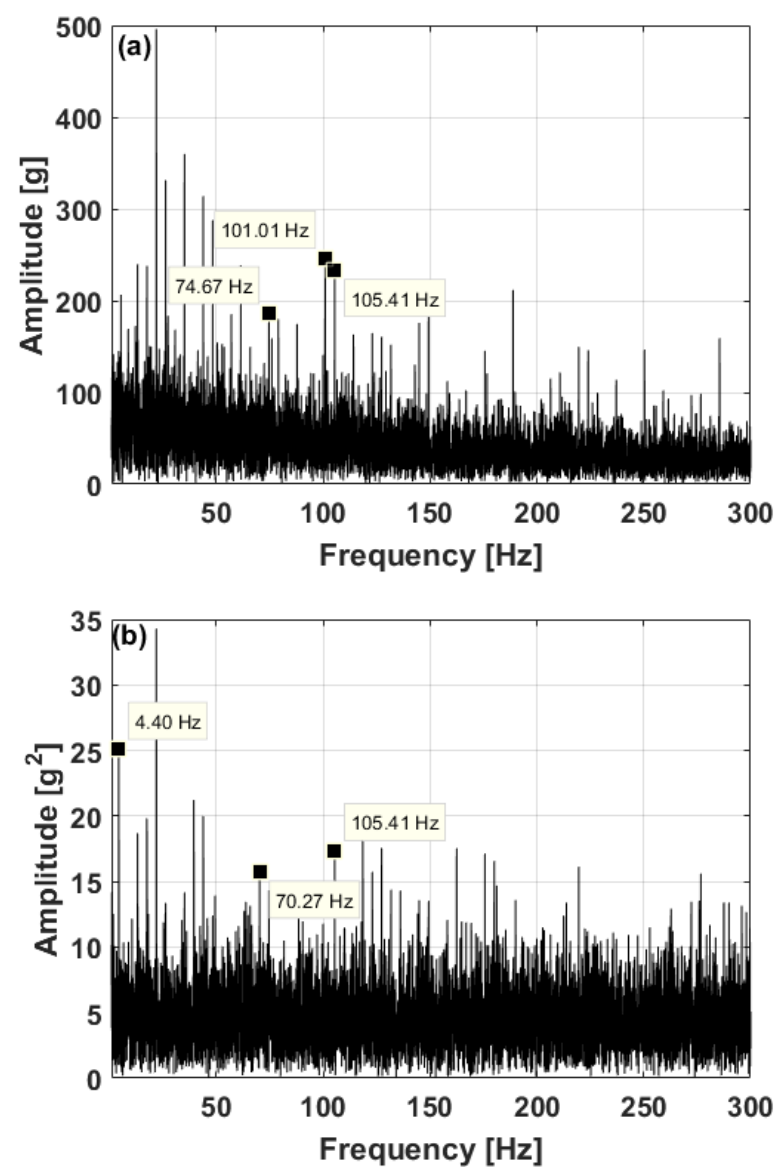

Figure 11: Squared envelope spectrum of Y6 data, processed using:

(a): SANC and iterative envelope cancellation

(b): Cepstrum editing, SANC and iterative envelope cancellation

The signal processing for Y7 data followed similar procedures. Cepstrum editing, DRS separation and iterative envelope cancellation techniques were applied to Y7 data. The results are shown in Figure 12. 

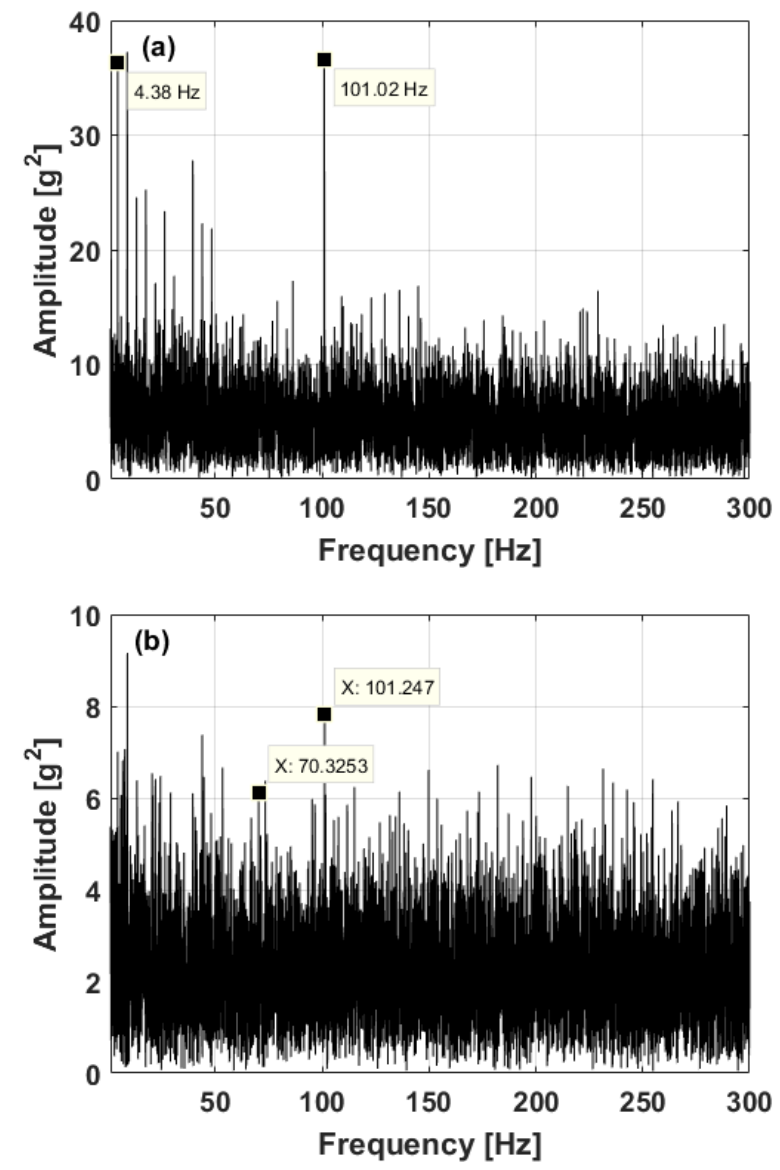

Figure 12: Squared envelope spectrum of Y7 data, processed using:

(a): DRS and iterative envelope cancellation

(b): Cepstrum editing, DRS and iterative envelope cancellation

In Figure 12(a), the diagnosis result is conclusive. The $101.02 \mathrm{~Hz} F_{I R D}$ was clearly evident, as well as the $4.4 \mathrm{~Hz}$ drive shaft speed, while the $F_{O R D}$ is still masked. Practically at this point, applying cepstrum editing could be redundant since the sidebands around $F_{I R D}$ are not dominant, but for the investigation of $F_{O R D}$, cepstrum editing is performed in Figure 12(b). Result proves that cepstrum editing may render some losses for inner race defect bearing signal, whereas the $70.3 \mathrm{~Hz} F_{O R D}$ is revealed.

Comparatively, squared envelope spectrum results produced using SANC as separation technique is demonstrated in Figure 13. Applying SANC without cepstrum for Y7 data can reveal the fault-related sideband $105.4 \mathrm{~Hz}$, but no direct indication of fault frequency can be observed for both $F_{I R D}$ and $F_{O R D}$. Moreover, applying cepstrum editing in Figure 13(b) tends to severely eliminate residual bearing signal under this circumstance. 

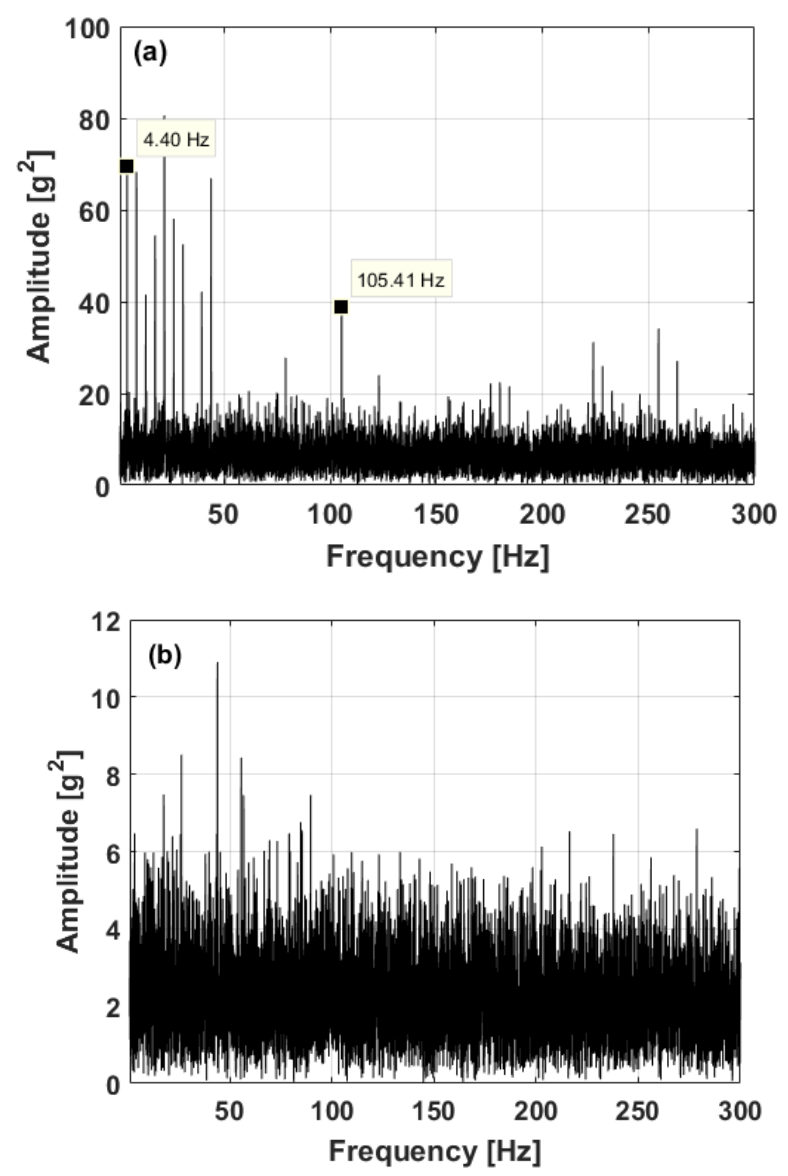

Figure 13: Squared envelope spectrum of $\mathrm{Y} 7$ data, processed using:

(a): SANC and iterative envelope cancellation

(b): Cepstrum editing, SANC and iterative envelope cancellation

Kurtogram combined with envelope spectrum analysis were implemented as a comparison to the optimal results demonstrated in Figure 8 and Figure 12. DRS filtered $\mathrm{Y} 6$ and $\mathrm{Y} 7$ data were adopted as inputs for kurtogram.

Kurtogram indication for Y6 data and corresponding squared envelope spectrum are shown in Figure 14. It is indicated that the optimal bandpass centre frequency is $12889.5 \mathrm{~Hz}$ with a bandwidth of $25779.02 \mathrm{~Hz}$. The demodulated squared envelope spectrum in Figure 14(b) contains distinct indication of $F_{I R D}$ near $101 \mathrm{~Hz}$ and $F_{O R D}$ near $70 \mathrm{~Hz}$, however, the sidebands and harmonics are excessive compared with result shown in Figure 8 using iterative envelope cancellation techniques. Similarly, processing result using kurtogram for Y7 data is shown in Figure 15, where fault frequencies can be identified, but the spectrum is also contaminated by excessive frequency sidebands and harmonics. 

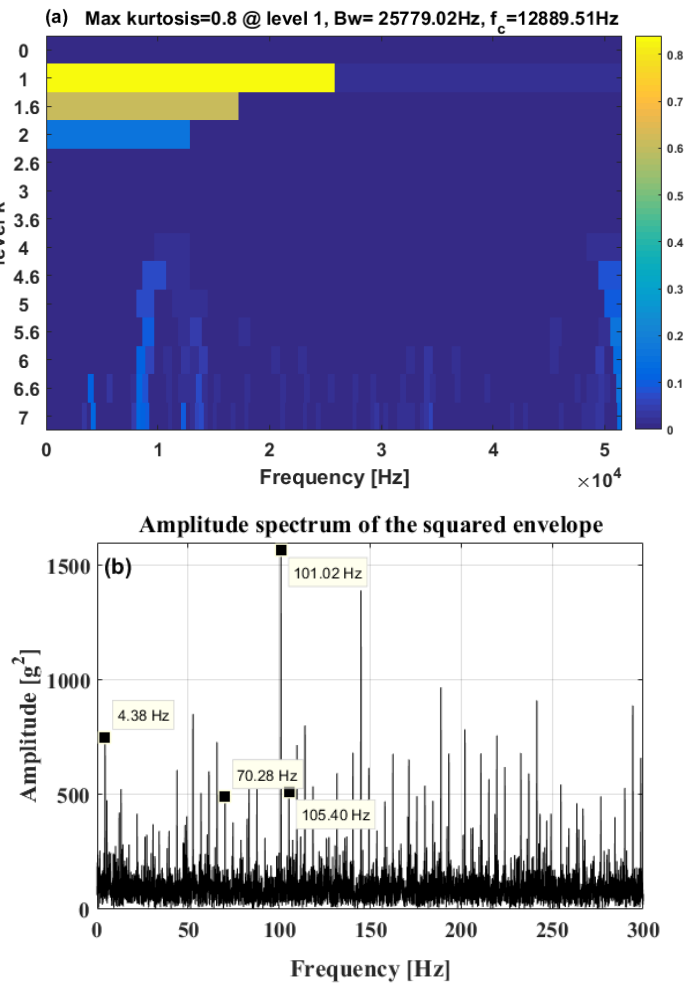

Figure 14: Kurtogram indication of DRS filtered Y6 data in (a); corresponding squared envelope spectrum in (b)

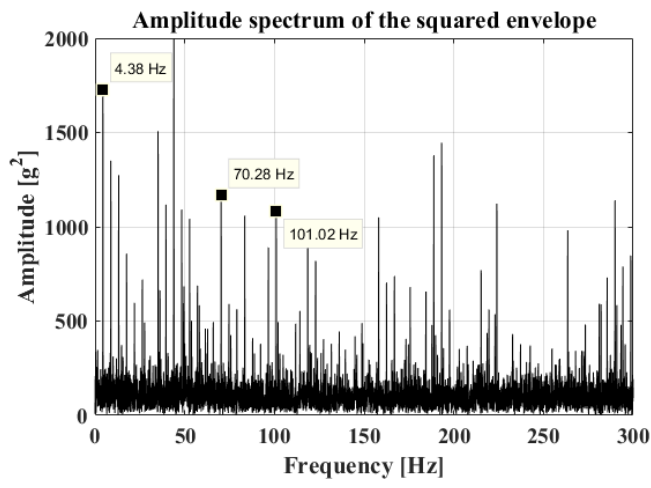

Figure 15: Squared envelope spectrum of DRS filtered Y7 data

\section{Discussion and conclusion}

In this paper, the helicopter planetary bearing inner race defect and outer race defect were successfully diagnosed for both $\mathrm{Y} 6$ and $\mathrm{Y} 7$ channel by imple- 
menting frequency domain analysis techniques including DRS, SANC, cepstrum editing, iterative envelope cancellation and kurtogram combined with envelope analysis. Decisive indications of fault-related frequency components can be directly observed in squared envelope spectrum shown in Figure 8 and Figure 12. Processing techniques were evaluated in terms of their efficacy and effectiveness. Regarding the $\mathrm{CH}-46 \mathrm{E}$ helicopter dataset, due to the high data sampling frequency $(103116.08 \mathrm{~Hz})$ and large data size, SANC is less efficient which is attributed to its adaptive process. Meanwhile, the DRS technique performs considerably well in combination with the iterative envelope cancellation technique, which is capable of suppressing residual gear mesh and frequency sidebands. Cepstrum editing can be adopted as an additional technique to deal with frequency sidebands, but it could also potentially hinder the weak bearing signal extraction, e.g. Y7 data in our case. Kurtogram has given reliable diagnosis results as a benchmark technique.

The successful extraction of frequency domain diagnostic information has produced intuitive indications for $\mathrm{CH}-46 \mathrm{E}$ helicopter planetary bearing defects. This study benefits not only the enhancement of HUMS performance, but also the improvement of accuracy for the established classification-based diagnosis algorithms. For future research, the authors would like to utilise the outcome of this study to achieve automated diagnosis process for helicopter gearbox planetary bearings.

\section{Reference}

[1] Federal Aviation Administration, Helicopter Flying Handbook, Chapter 04: Helicopter Components, Sections, and Systems. [Online]. Available: www.faa.gov/regulations policies/handbooks manuals

[2] J. E. Land, "Hums-the benefits-past, present and future," in Aerospace Conference, 2001, IEEE Proceedings., vol. 6. IEEE, 2001, pp. 30833094.

[3] Joint Helicopter Safety Implementation Team, "Health and usage monitoring systems toolkit," 2013.

[4] R. Hess, A. Duke, and D. Kogut, "The IMD HUMS as a tool for rotorcraft health management and diagnostics," in Aerospace Conference, 2001, IEEE Proceedings., vol. 6. IEEE, 2001, pp. 3039-3058.

[5] Civil Aviation Authority, "Cap 1145-safety review of offshore public transport helicopter operations in support of the exploitation of oil and gas," 2014. 
[6] K. Fraser, "An overview of health and usage monitoring systems (HUMS) for military helicopters," Tech. Rep., 1994.

[7] B. D. Rex, "Non-invasive detection of CH-46 aft gearbox faults using digital pattern recognition and classification techniques," Naval Aacademy Annapolis MD, Tech. Rep., 1999.

[8] AIBN, "Accident at Turoy, near Bergen, Norway on 29 April 2016, involving Airbus helicopters H225, LN-OJF," Accident Investigation Board Norway, Tech. Rep., 2016.

[9] Air Accidents Investigation Branch, "Report on the accident to Aerospatiale Eurocopter AS332 L2 super puma, registration G-REDL $11 \mathrm{~nm}$ NE of Peterhead, Scotland on 1 April 2009," Air Accidents Investigation Branch, Tech. Rep., 2009.

[10] K. Pipe, "Measuring the performance of a hum system-the features that count," in Third international conference on health and usage monitoring-HUMS2003, 2002, p. 5.

[11] M. Greaves, F. Elasha, J. Worskett, D. Mba, H. Rashid, and R. Keong, "Vhm: Vibration health or alternative monitoring technologies for helicopters," European Aviation Safety Agency, Tech. Rep., 2012.

[12] B. G. Cameron, "Final report on CH-46 aft transmission seeded fault testing," Westland Helicopters Ltd, UK, Research Paper RP90\%, 1993.

[13] Isotron accelerometer product specification, isotron accelerometer model 7259a-10, -25 and -100 . [Online]. Available: http://www.ic72.com/pdf'file/7/544158.pdf

[14] A. Aviation and M. L. C. Command, "Ads-79b-hdbk (2011), handbook for condition based maintenance systems for us army aircrafts," 2011.

[15] R. B. Randall, Vibration-based condition monitoring: industrial, aerospace and automotive applications. John Wiley \& Sons, 2011.

[16] J. Antoni and R. Randall, "Unsupervised noise cancellation for vibration signals: part I-evaluation of adaptive algorithms," Mechanical Systems and Signal Processing, vol. 18, no. 1, pp. 89-101, 2004.

[17] F. Bonnardot, R. B. Randall, J. Antoni, and F. Guillet, "Enhanced unsupervised noise cancellation (E-SANC) using angular resampling application for planetary bearing fault diagnosis," Surveillance, vol. 5, pp. 11-13, 2004. 
[18] J. Antoni and R. Randall, "Unsupervised noise cancellation for vibration signals: part II-a novel frequency-domain algorithm," Mechanical Systems and Signal Processing, vol. 18, no. 1, pp. 103-117, 2004.

[19] J. Antoni, "The spectral kurtosis: a useful tool for characterising nonstationary signals," Mechanical Systems and Signal Processing, vol. 20, no. 2, pp. 282-307, 2006.

[20] J. Antoni and R. Randall, "The spectral kurtosis: application to the vibratory surveillance and diagnostics of rotating machines," Mechanical Systems and Signal Processing, vol. 20, no. 2, pp. 308-331, 2006.

[21] J. Antoni, "Fast computation of the kurtogram for the detection of transient faults," Mechanical Systems and Signal Processing, vol. 21, no. 1, pp. 108-124, 2007.

[22] R. B. Randall and J. Antoni, "Rolling element bearing diagnostics-a tutorial," Mechanical systems and signal processing, vol. 25, no. 2, pp. 485-520, 2011.

[23] D. Ho and R. Randall, "Effects of time delay, order of fir filter and convergence factor on self-adaptive noise cancellation," in International Conference on Sound and Vibration (ICSV5), Adelaide, 1997, pp.--.

[24] B. Widrow, J. R. Glover, J. M. McCool, J. Kaunitz, C. S. Williams, R. H. Hearn, J. R. Zeidler, J. E. Dong, and R. C. Goodlin, "Adaptive noise cancelling: Principles and applications," Proceedings of the IEEE, vol. 63, no. 12, pp. 1692-1716, 1975.

[25] D. Ho and R. Randall, "Optimisation of bearing diagnostic techniques using simulated and actual bearing fault signals," Mechanical systems and signal processing, vol. 14, no. 5, pp. 763-788, 2000.

[26] C. Ruiz-Carcel, E. Hernani-Ros, P. Chandra, Y. Cao, and D. Mba, "Application of linear prediction, self-adaptive noise cancellation, and spectral kurtosis in identifying natural damage of rolling element bearing in a gearbox," in Proceedings of the 7th World Congress on Engineering Asset Management (WCEAM 2012). Springer, 2015, pp. 505-513.

[27] F. Elasha, C. Ruiz-Carcel, D. Mba, and P. Chandra, "A comparative study of the effectiveness of adaptive filter algorithms, spectral kurtosis and linear prediction in detection of a naturally degraded bearing in a gearbox," Journal of Failure Analysis and Prevention, vol. 14, no. 5, pp. 623-636, 2014. 
[28] N. Sawalhi and R. Randall, "Localized fault detection and diagnosis in rolling element bearings: A collection of the state of art processing algorithms." no. Hums, 2013.

[29] T. Barszcz, "Decomposition of vibration signals into deterministic and nondeterministic components and its capabilities of fault detection and identification," International Journal of Applied Mathematics and Computer Science, vol. 19, no. 2, pp. 327-335, 2009.

[30] R. B. Randall, "A history of cepstrum analysis and its application to mechanical problems," Mechanical Systems and Signal Processing, 2016.

[31] R. Randall, B. Peeters, J. Antoni, and S. Manzato, "New cepstral methods of signal preprocessing for operational modal analysis," in Proc. Int. Conference on Noise and Vibration Engineering (ISMA), 2012, pp. -.

[32] R. Randall, N. Sawalhi, and M. Coats, "A comparison of methods for separation of deterministic and random signals," International Journal of Condition Monitoring, vol. 1, no. 1, pp. 11-19, 2011.

[33] N. Sawalhi and R. Randall, "Signal pre-whitening using cepstrum editing (liftering) to enhance fault detection in rolling element bearings," in Proceedings of the 24 international congress on condition monitoring and diagnostic engineering management (comadem2011), may, 2011, pp. 330-336.

[34] R. Randall and W. Smith, "New cepstral methods for the diagnosis of gear and bearing faults under variable speed conditions," in ICSV23 conference, Athens, 2016.

[35] P. Borghesani, P. Pennacchi, R. Randall, N. Sawalhi, and R. Ricci, "Application of cepstrum pre-whitening for the diagnosis of bearing faults under variable speed conditions," Mechanical Systems and Signal Processing, vol. 36, no. 2, pp. 370-384, 2013.

[36] M. S. Darlow, R. H. Badgley, and G. Hogg, "Application of highfrequency resonance techniques for bearing diagnostics in helicopter gearboxes." Tech. Rep., 1974.

[37] A. Ming, W. Zhang, Z. Qin, and F. Chu, "Envelope calculation of the multi-component signal and its application to the deterministic component cancellation in bearing fault diagnosis," Mechanical Systems and Signal Processing, vol. 50, pp. 70-100, 2015. 
[38] R. Dwyer, "Use of the kurtosis statistic in the frequency domain as an aid in detecting random signals," IEEE Journal of Oceanic Engineering, vol. 9, no. 2, pp. 85-92, 1984.

[39] V. Vrabie, P. Granjon, and C. Serviere, "Spectral kurtosis: from definition to application," in 6th IEEE International Workshop on Nonlinear Signal and Image Processing (NSIP 2003), 2003.

[40] X. Tian, J. X. Gu, I. Rehab, G. M. Abdalla, F. Gu, and A. D. Ball, "A robust detector for rolling element bearing condition monitoring based on the modulation signal bispectrum and its performance evaluation against the kurtogram," Mechanical Systems and Signal Processing, vol. 100, pp. 167-187, 2018. 